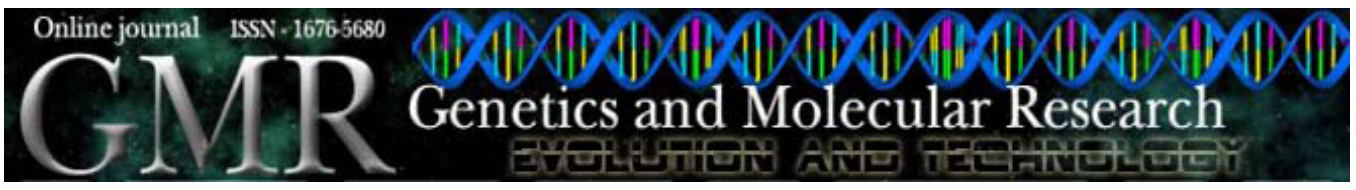

Case Report

\title{
A small supernumerary marker chromosome, derived from chromosome 22, possibly associated with repeated spontaneous abortions
}

\author{
M. Balkan', H. İsi', A. Gedik², M. Erdemoğlu ${ }^{3}$ and T. Budak \\ ${ }^{1}$ Department of Medical Biology and Genetic, Medical Faculty, \\ Dicle University, Diyarbakır, Turkey \\ ${ }^{2}$ Department of Urology, Medical Faculty, \\ Dicle University, Diyarbakır, Turkey \\ ${ }^{3}$ Department of Gynecology and Obstetrics, Medical Faculty, \\ Dicle University, Diyarbakır, Turkey \\ Corresponding author: M. Balkan \\ E-mail: balkanmah@gmail.com /mahbal@dicle.edu.tr
}

Genet. Mol. Res. 9 (3): 1683-1689 (2010)

Received May 10, 2010

Accepted June 20, 2010

Published August 24, 2010

DOI 10.4238/vol9-3gmr947

\begin{abstract}
We report a phenotypically normal couple with repeated spontaneous abortions and without other clinical features. Clinical, hematological, biochemical, and endocrinological aspects of the couple did not reveal any abnormalities. The karyotype of the wife was normal $(46, X X)$, while the husband was found to have an abnormal karyotype, 47,XY,+der(22)mat. The marker chromosome was familial and non-satellite. Although the potential risk of small supernumerary marker chromosomes for spontaneous abortions cannot be defined precisely, marker chromosomes, together with methods used for ascertainment, are also factors to be considered when investigating infertility consequences. Furthermore, identification of the origin of a marker chromosome may provide additional information for patient karyotype-phenotype correlations. Further studies, such as molecular analyses to identify the breakpoint, are necessary for investigating
\end{abstract}


phenotype-genotype correlations and assessment of genetic risks for small secondary chromosomes. The cause of repeated spontaneous abortions in this couple might be the presence of this marker chromosome in the husband. Consequently, we recommended genetic counseling before further pregnancies.

Key words: Small supernumerary marker chromosomes; Karyotype; Spontaneous abortion; Marker chromosome

\section{INTRODUCTION}

Fertility problems and/or infertility are common phenomenons, and their presumptive causes include anatomical, endocrinological and immunological factors, infections, thrombophilic disorders, and other unexplained causes (Diego-Alvarez et al., 2006; Manvelyan et al., 2008). In about $20 \%$ of infertile couples, the etiology remains 'unexplained'. Most, if not all, the aforementioned factors of infertility are likely to have genetic components, such as deleterious gene mutations and microdeletions and the presence of constitutional, numerical and structural aberrations of chromosomes (Manvelyan et al., 2008). Cytogenetic abnormalities are well established as an important cause of both sporadic and recurrent spontaneous abortions (Blumberg et al., 1982). More than 50\% of spontaneous miscarriages carry chromosomal disorders, up to $96 \%$ of which are numerical chromosomal abnormalities consisting of the presence of small supernumerary marker chromosomes (sSMCs), sex chromosome aberrations and constitutional aberrations such as inversions and translocations (Diego-Alvarez et al., 2006; Manvelyan et al, 2008). In a recent study, the proportion of individuals having sSMCs in the normal population was reported as $0.044 \%$, while the proportion was as high as $0.125 \%$ in infertile groups (Manvelyan et al., 2008).

Although the potential risk of an sSMC for fetal loss cannot be defined precisely, and it has not even been ascertained whether the association of an SSMC to fetal loss is causal or coincidental, the sSMC, together with methods used for ascertainment, are also factors to be considered when estimating fertility consequences. Furthermore, identification of the origin of an sSMC may provide additional information for karyotype-phenotype correlations (Kumar et al. 1997). In addition, the limited data available do not permit a reliable phenotypegenotype correlation. Here we report on a couple whose husband had a marker chromosome and karyotype 47,XY,+der(22)mat and his wife had four spontaneous miscarriages, one baby with neonatal death, and only one healthy birth. The father's mother, who also carried the marker chromosome, declared 14 known pregnancies, with 5 ending in miscarriage and two in neonatal death.

\section{CASE REPORT}

A young, healthy and non-consanguineous couple was referred to our department for genetic evaluation due to spontaneous abortions. The couple had natural conceptions ending in four spontaneous abortions and one neonatal death, as well as a healthy girl. The husband was 35 years old, and his wife was 26 years old. In the urological and gynecological investigations including anatomical, endocrinological, infectious, and immunological 
tests, they were found to be phenotypically normal. The couple was analyzed for chromosomal abnormalities. The family history was unremarkable, except for infertility in a sister of the husband and hemophilia in his mother and brothers. Chromosomal analysis in the couple and other family members was carried out on peripheral blood lymphocyte culture by using the standard protocol of Seabright (1971), with slight modifications. Chromosomes were examined by GTG banding, and metaphases were karyotyped on a microscope equipped with a CCD camera and an image analysis system (MetaSystems, Altlussheim, Germany). We analyzed a total of 50 cells in each subject to exclude any subtle mosaicism for sSMCs (Liehr, 2006). The karyotypic descriptions were reported according to the International System for Human Cytogenetic Nomenclature recommendations (ISCN, 1995). Additionally, the chromosomes were stained with Ag-AS for nucleolus organizer region (NOR) analysis in accordance with a modification of the method of Bloom and Goodpasture (1976) to reveal NOR (satellite).

Fluorescence in situ hybridization (FISH) studies were performed according to the protocols provided by the manufacturer (Cytocell for whole-chromosome painting), using indirectly labeled octoChrome painting probes (Chromoprobe Multiprobe System OctoChrome, Cytocell). The results were evaluated on a fluorescence microscope equipped with a CCD camera and an image analysis system (MetaSystems). Cytocell's Chromoprobe Multiprobe System OctoChrome combines the utility of an 8-square Multiprobe device and whole chromosome painting probes, labeled in 3 different colors, to allow the simultaneous analysis of all 24 chromosomes on one slide in a single hybridization. Each square of the Multiprobe device carries the whole chromosome by painting probes for three different chromosomes in three different color fluorophores, red, green and blue (Texas Red, FITC and Coumarin spectra, respectively), which are visible simultaneously with a DAPI/FITC/Texas Red triple filter. The results were evaluated with the same fluorescence microscope.

This study was performed in accordance with the principles of the Declarations of Helsinki. Participants were informed of the nature of the study, and written consent was obtained from the couple for the publication of this study.

\section{RESULTS}

Clinical, hematological, biochemical, and endocrinological investigations on the couple did not reveal any abnormality. The chromosomal analysis with conventional G-banding revealed that the karyotype of the wife was normal $(46, \mathrm{XX})$. However, an extra small marker chromosome was observed in all the 50 cells analyzed from the husband (Figure 1A). FISH analysis by using all 24 whole-chromosome paints identified that the marker chromosome was derived from chromosome 22, and all the metaphase plates were with the marker chromosome 22 , suggesting that he was nonmosaic 47,XY,+mar (Figure 1B). G-banding and FISH studies revealed that his karyotype was $47, \mathrm{XY},+\operatorname{der}(22)$ mat. Additionally, by the silver-staining method for satellite material, the sSMC found was non-satellite (Figure 1C). Then, his parents were investigated cytogenetically to know the parental origin of the marker chromosome. While his father $(46, \mathrm{XY})$ was normal, the mother was found to carry the marker chromosome $(47, \mathrm{XX},+$ mar22), demonstrating that marker chromosome in the proband was not de novo but inherited. The mother's obstetric history was poor, with fourteen known pregnancies ending in five second-trimester spontaneous abortions, two neonatal deaths and seven healthy children. 
A


B



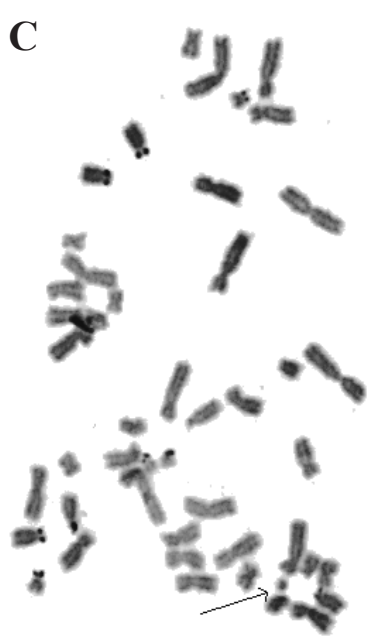

Figure 1. A. Cytogenetic characterization of the marker chromosome. Arrows indicate marker chromosome. B. Molecular cytogenetic characterization of the marker chromosome. FISH whole chromosome painting (WCP) analysis. WCPs 5 (blue), 9 (red), and 22 (green) applied to metaphase chromosomes. Arrow indicates marker chromosome. C. Silver or NOR staining of the marker chromosome. Arrow indicates non-satellited marker chromosome.

\section{DISCUSSION}

Evaluation of a patient with a history of repeated spontaneous abortions requires careful consideration of potential genetic, anatomical, endocrinological, infectious, and immunological risk factors (Diego-Alvarez et al., 2006). Except for a genetic factor in the 
husband, no risk was found in the couple who we studied.

In order to arrive at an accurate diagnosis for a genetic condition, conventional cytogenetics need to be supported with molecular investigations (Kumar et al., 1997; DiegoAlvarez et al., 2006; Graf et al., 2006). Cytogenetic investigation by the conventional Gbanding method in the husband revealed a small metacentric chromosome, which was further confirmed as marker chromosome 22 by the FISH technique.

The risk for phenotypic abnormalities associated with an SSMC depends on several factors, including inheritance, chromosomal origin, content, and structure of the marker (Graf et al., 2006). About $70 \%$ of sSMCs derive from acrocentric chromosomes, and sSMCs derived from chromosome 22 comprise $18.6 \%$ of the acrocentric cases (Liehr et al., 2004). The phenotypes associated with the presence of a marker vary from normal to severely abnormal (Paoloni-Giacobino et al., 1998). In general, 70\% of carriers of sSMCs derived from chromosome 22 are clinically normal (http://www.med.uni-jena.de/fish/sSMC/00START.htm). In our case, the sSMC was also derived from chromosome 22 and a normal phenotype was observed. Although some studies provided indications concerning the pathologic phenotype and the risk accordingly associated with an sSMC (Warburton, 1991; Crolla, 1998), the published data still do not allow any definite conclusions to be drawn concerning karyotype-phenotype correlations (Dalprà et al., 2005).

It has been reported that $44 \%$ of prenatally ascertained cases with sSMCs are familial (http://www.med.uni-jena.de/fish/sSMC/00START.htm.). In some families, sSMCs are transmitted through several generations, apparently with no associated abnormalities, whereas other sSMC carriers may present serious clinical symptoms, such as mental retardation, dysmorphic features and malformations (Liehr et al., 2004). Ridler et al. (1970) described the first sSMC carriers without clinical abnormalities in three generations [sSMC-database (http:// markerchromosomes.ag.vu or http://www.med.uni-jena.de/fish/sSMC/00START.htm)]. In our case, the SSMC was of maternal origin, apparently with no associated abnormalities in either the proband or his mother. However, there are exceptions to this, such as when there are imprinting effects from uniparental disomy, or low level, tissue specific mosaicism for the sSMC in a parent without phenotypic manifestations (Graf et al., 2006).

sSMCs are also classified as satellited or non-satellited and mosaic or non-mosaic (Crolla et al., 2005). It has previously been reported that carrying an accessory bisatellited sSMC was not a risk factor for having abnormal offspring, spontaneous abortion or nondisjunction (Kumar et al., 1997). Warburton (1984) and Djalali (1990) observed that 7\% of sSMCs resulting in phenotypically normal fetuses had satellites. There is some evidence suggesting the influence of both non-satellited and satellited sSMCs on having abnormal offspring, spontaneous abortion or non-disjunction (Warburton, 1991). The risk of phenotypic abnormality due to non-satellited and satellited sSMC was reported as 14.7 and $10.9 \%$, respectively (Kumar et al., 1997). In our study, the case was non-satellited and non-mosaic, and the possible explanation for four spontaneous abortions in the proband's wife and five in his mother in our study family is likely because the sSMC was non-satellite.

The mechanism by which sSMCs influence fertility has not yet been understood (Manvelyan et al., 2008). It has been suggested that sSMCs may lead to reduced fertility in males without additional clinical symptoms in connection with the sSMC (Mulcahy and Jenkyn, 1972; Chandley et al., 1975; Mau et al., 1997; Manvelyan et al., 2008). According to population studies, sSMCs are found approximately 2.9 times more often in healthy persons with un- 
explained infertility compared to the general population (Manvelyan et al., 2008). Manvelyan et al. (2008) reported that an enhanced rate of repeated abortions in sSMC carriers or their partners was observed in $22-35 \%$ of the cases. In another report, of the 123 cases with sSMCs detected prenatally, 37 were electively terminated, and only 4 of the remaining 86 pregnancies ended with a still birth or spontaneous abortion. Paoloni-Giacobino et al. (1998) reported that nine additional cases of 86 cases were born with abnormalities (10.5\%). According to Kumar et al. (1997), 4.4\% of sSMC pregnancies end in stillbirth or spontaneous abortion. However, we hypothesized that perhaps additional genetic factors in the family contributed in concert with sSMCs to produce the high frequency of miscarriages.

Some reports show that familial sSMCs are predominantly inherited via the maternal line (Warburton, 1991; Liehr, 2006; Manvelyan et al., 2008). Liehr (2006) suggested that a yet unknown effect is driving selection via the fertilization success of sperm without an SSMC. At least two possible mechanisms could be proposed to explain this phenomenon: Problems in connection with SSMC-replication arising predominantly in the more rapidly progressing sperm meiosis or a 'weight-effect', that is, the sSMC slowing down the sperm, similar to the effect known from Y- versus X-chromosome carrying sperm (Manvelyan et al., 2008). In our case, the familial sSMC was inherited via the maternal line; this observation is consistent with the recently outlined fact that familial sSMCs are predominantly inherited via the maternal line (Warburton, 1991; Liehr, 2006; Manvelyan et al., 2008).

It was also reported that there appeared to be gender-specific differences for the children: a maternally inherited SSMC was more likely to be detected in a son with fertility problems than in daughters and vice versa for paternally inherited sSMCs (Manvelyan et al., 2008). Our findings were consistent with the report.

Consequently, in our case, the behavior of $\operatorname{mar}(22)$ in relation to fetal loss in his partnership has been a subject of scrutiny and debate. Although no chromosome analysis has been performed on the aborted fetuses, we propose that the possible explanation for the repeated spontaneous abortions in our case is likely due to the presence of marker chromosomes. Therefore, we recommended genetic counseling before further pregnancies.

\section{REFERENCES}

Bloom SE and Goodpasture C (1976). An improved technique for selective silver staining of nucleolar organizer regions in human chromosomes. Hum. Genet. 34: 199-206.

Blumberg BD, Shulkin JD, Rotter JI, Mohandas T, et al. (1982). Minor chromosomal variants and major chromosomal anomalies in couples with recurrent abortion. Am. J. Hum. Genet. 34: 948-960.

Chandley AC, Edmond P, Christie S, Gowans L, et al. (1975). Cytogenetics and infertility in man. I. Karyotype and seminal analysis: results of a five-year survey of men attending a subfertility clinic. Ann. Hum. Genet. 39: 231-254.

Crolla JA (1998). FISH and molecular studies of autosomal supernumerary marker chromosomes excluding those derived from chromosome 15: II. Review of the literature. Am. J. Med. Genet. 75: 367-381.

Crolla JA, Youings SA, Ennis S and Jacobs PA (2005). Supernumerary marker chromosomes in man: parental origin, mosaicism and maternal age revisited. Eur. J. Hum. Genet. 13: 154-160.

Dalprà L, Giardino D, Finelli P, Corti C, et al. (2005). Cytogenetic and molecular evaluation of 241 small supernumerary marker chromosomes: cooperative study of 19 Italian laboratories. Genet. Med. 7: 620-625.

Diego-Alvarez D, Ramos-Corrales C, Garcia-Hoyos M, Bustamante-Aragones A, et al. (2006). Double trisomy in spontaneous miscarriages: cytogenetic and molecular approach. Hum. Reprod. 21: 958-966.

Djalali M (1990). The significance of accessory bisatellited marker chromosomes in amniotic fluid cell cultures. Ann. Genet. 33: 141-145.

Graf MD, Christ L, Mascarello JT, Mowrey P, et al. (2006). Redefining the risks of prenatally ascertained supernumerary 
marker chromosomes: a collaborative study. J. Med. Genet. 43: 660-664.

International Software Consulting Network (ISCN) (1995). An International System for Human Cytogenetic Nomenclature.

In: Recommendations of the International Standing Committee on Human Cytogenetic Nomenclature (Mitelman F, ed.). S. Karger Publishers Inc., Basel.

Kumar C, Svetlana MK, Rhea VS and Ram SV (1997). Marker chromosomes in fetal loss. Hum. Reprod. 12: 1321-1324. Liehr T (2006). Familial small supernumerary marker chromosomes are predominantly inherited via the maternal line. Genet. Med. 8: 459-462.

Liehr T, Claussen U and Starke H (2004). Small supernumerary marker chromosomes (sSMC) in humans. Cytogenet. Genome Res. 107: 55-67.

Manvelyan M, Riegel M, Santos M, Fuster C, et al. (2008). Thirty-two new cases with small supernumerary marker chromosomes detected in connection with fertility problems: detailed molecular cytogenetic characterization and review of the literature. Int. J. Mol. Med. 21: 705-714.

Mau UA, Backert IT, Kaiser P and Kiesel L (1997). Chromosomal findings in 150 couples referred for genetic counselling prior to intracytoplasmic sperm injection. Hum. Reprod. 12: 930-937.

Mulcahy MT and Jenkyn J (1972). Results of 538 chromosome studies on patients referred for cytogenetic analysis. Med. J. Aust. 2: 1333-1338.

Paoloni-Giacobino A, Morris MA and Dahoun SP (1998). Prenatal supernumerary r(16) chromosome characterized by multiprobe FISH with normal pregnancy outcome. Prenat. Diagn. 18: 751-752.

Ridler MA, Berg JM, Pendrey MJ, Saldaña P, et al. (1970). Familial occurrence of a small, supernumerary metacentric chromosome in phenotypically normal women. J. Med. Genet. 7: 148-152.

Seabright M (1971). A rapid banding technique for human chromosomes. Lancet 2: 971-972.

Warburton D (1984). Outcome of de novo structural rearrangements diagnosed translocation heterozygotes at amniocentesis as determined by potential at amniocentesis. Prenat. Diagn. 4 (Special Issue): 69-80.

Warburton D (1991). De novo balanced chromosome rearrangements and extra marker chromosomes identified at prenatal diagnosis: clinical significance and distribution of breakpoints. Am. J. Hum. Genet. 49: 995-1013. 